\title{
COMPACT-LIKE TOTALLY DENSE SUBGROUPS OF COMPACT GROUPS
}

\author{
DIKRAN N. DIKRANJAN AND DMITRII B. SHAKHMATOV
}

(Communicated by Dennis Burke)

\begin{abstract}
A subgroup $H$ of a topological group $G$ is (weakly) totally dense in $G$ if for each closed (normal) subgroup $N$ of $G$ the set $H \cap N$ is dense in $N$. We show that no compact (or more generally, $\omega$-bounded) group contains a proper, totally dense, countably compact subgroup. This yields that a countably compact Abelian group $G$ is compact if and only if each continuous homomorphism $\pi: G \rightarrow H$ of $G$ onto a topological group $H$ is open. Here "Abelian" cannot be dropped. A connected, compact group contains a proper, weakly totally dense, countably compact subgroup if and only if its center is not a $G_{\delta}$-subgroup. If a topological group contains a proper, totally dense, pseudocompact subgroup, then none of its closed, normal $G_{\delta}$-subgroups is torsion. Under Lusin's hypothesis $2^{\omega}{ }_{1}=2^{\omega}$ the converse is true for a compact Abelian group $G$. If $G$ is a compact Abelian group with nonmetrizable connected component of zero, then there are a dense, countably compact subgroup $K$ of $G$ and a proper, totally dense subgroup $H$ of $G$ with $K \subseteq H$ (in particular, $H$ is pseudocompact).
\end{abstract}

\section{RESULtS}

All topological groups considered in this paper are assumed to be Hausdorff.

1.1. Definition [13]. We say that a subgroup $H$ of a topological group $G$ is (weakly) totally dense in $G$ provided that, for each closed (normal) subgroup $N$ of $G$, the set $H \cap N$ is dense in $N$.

It is clear that $H$ as above is dense in $G$ and that both notions coincide in case $G$ is Abelian. The notion of a totally dense subgroup was extensively investigated in the literature $[2,3,4,5,6,7,8,12]$. Its significance became clear in the context of the open mapping theory in the following sense.

\subsection{Definition [6]. A topological group $G$ is said to be totally minimal if each}

Received by the editors April 26, 1990 and, in revised form, October 26, 1990.

1980 Mathematics Subject Classification (1985 Revision). Primary 54D25; Secondary 22C05, $54 \mathrm{~A} 35$.

Key words and phrases. Compact group, countably compact group, pseudocompact group, totally minimal group, totally dense subgroup, $G_{\delta}$-subgroup, Lusin's hypothesis.

This work was done while the second author was visiting the Institute of Mathematics of the Bulgarian Academy of Sciences in March 1990, he would like to express his thanks to the Institute for their hospitality and support. 
continuous homomorphism $\pi: G \rightarrow H$ of $G$ onto a topological group $H$ is open.

The following result clarifies the interrelation between the notions defined above.

1.3. Total minimality criterion ([6], see also [7, 4.3.3]). A dense subgroup $H$ of a compact topological group $G$ is totally minimal if and only if $H$ is weakly totally dense in $G$.

In this paper we investigate the question whether a compact group contains a proper, (weakly) totally dense subgroup with additional "compact-like" properties such as countable compactness and pseudocompactness. Recall that a topological space $X$ is:

$\omega$-bounded if every countable subset of $X$ has compact closure;

countably compact if every infinite subset of $X$ has an accumulation point in $X$;

pseudocompact if every real-valued continuous function defined on $X$ is bounded.

It is well known that compact $\rightarrow \omega$-bounded $\rightarrow$ countably compact $\rightarrow$ pseudocompact, but neither of these implications is reversible (see [9]).

In 1982 Comfort and Soundararajan proved that any countably compact totally dense subgroup of a totally disconnected compact Abelian group $G$ coincides with $G$ [5, Theorem 6.7]. Our first result shows that both "Abelian" and "totally disconnected" are superfluous here.

1.4. Theorem. No $\omega$-bounded (in particular, compact) group contains a proper, totally dense, countably compact subgroup.

This theorem also substantially strengthens Corollary 6.8 of [5]. It should be noted that a general topological (Abelian) group can contain a proper, totally dense, countably compact subgroup. We give a detailed exposition of this phenomenon in a forthcoming paper.

Obviously, compact groups are countably compact and totally minimal. For Abelian groups the converse is also true.

1.5. Corollary. A countably compact, totally minimal Abelian group is compact. Proof. Let $G$ be a countably compact, totally minimal Abelian group. Since $G$ is pseudocompact, the completion $\widehat{G}$ of $G$ is compact [1, Theorem 6.3 and 1.13]. By $1.3, G$ is totally dense in $\widehat{G}$, and Theorem 1.4 implies $G=\widehat{G}$.

1.6. Corollary. If $G$ is a countably compact, noncompact Abelian group, then there exists a continuous homomorphism $\pi: G \rightarrow H$ of $G$ onto a topological group $H$ that is not open.

Comfort and Grant [2] and Guran [10] found out examples of totally minimal, countably compact, noncompact (necessarily non-Abelian) groups. These examples show that "Abelian" cannot be dropped in Corollaries 1.5 and 1.6.

Now let us consider the pseudocompact case. Here there is an easy necessary condition for a topological group to contain a proper, totally dense, pseudocompact subgroup.

1.7. Theorem. Let $G$ be a topological group that has a torsion, closed, normal $G_{\delta}$-subgroup $N$. Then $G$ contains no proper, totally dense, pseudocompact subgroup. 
Proof. Let $H$ be a totally dense, pseudocompact subgroup of $G$. First observe that $N \subseteq H$. Indeed, pick $x \in N$. Since $N$ is torsion, the smallest subgroup $K$ of $G$ containing $x$ is finite. Since $G$ is Hausdorff, $K$ is a closed discrete subgroup of $G$. Therefore $x \in K=H \cap K \subseteq H$ because $H$ is totally dense in $G$. Further, let $N=\bigcap\left\{V_{n}: n \in \mathbb{N}\right\}$, where all $V_{n}$ 's are open in $G$. We can construct by induction a sequence $\left\{U_{n}: n \in \mathbb{N}\right\}$ of open neighbourhoods of the neutral element of $G$ so that $U_{n}^{-1}=U_{n}$ and $U_{n+1}^{2} \subseteq U_{n} \cap V_{n}$ for all $n \in \mathbb{N}$. Then $N^{\prime}=\bigcap\left\{U_{n}: n \in \mathbb{N}\right\} \subseteq \bigcap\left\{V_{n}: n \in \mathbb{N}\right\}=N$ is a closed subgroup of $G$. Let $\varphi: G \rightarrow G / N^{\prime}$ be the natural quotient map from $G$ onto the left coset space $G / N^{\prime}$. From $[11,8.2]$ it follows that $G / N^{\prime}$ is submetrizable, i.e. there exists a one-to-one continuous map $i: G / N^{\prime} \rightarrow M$ onto a metric space $M$. Observe that $H^{*}=i(\varphi(H))$ is a dense, pseudocompact subspace of the metric space $M$. Since pseudocompact metric spaces are compact $[9,8.5 .13(\mathrm{c})], H^{*}=M$. Since $N^{\prime} \subseteq N$, we conclude that $H \cap(g+N) \neq \varnothing$ for every $g \in G$. Therefore

$$
H=H+H \supseteq H+N=G \text {. }
$$

It turns out that the condition described in Theorem 1.7 is the unique constraint for the existence of proper, totally dense, pseudocompact subgroups of compact Abelian groups. However, we were able to prove this only under Lusin's hypothesis $2^{\omega_{1}}=2^{\omega}$. This additional set-theoretic assumption is known to be consistent with the usual Zermelo-Fraenkel axioms ZFC of set theory [12].

1.8. Theorem. Assume Lusin's hypothesis $2^{\omega_{1}}=2^{\omega}$. Then the following conditions are equivalent for a compact Abelian group $G$ :

(i) $G$ contains a proper, totally dense, pseudocompact subgroup; and

(ii) no closed $G_{\delta}$-subgroup of $G$ is torsion.

In special cases we can construct totally dense, pseudocompact subgroups of compact Abelian groups without additional set-theoretical assumptions. Our next theorem improves Theorem 5.3 of [5]. The reader might compare it with Theorem 1.4.

1.9. Theorem. Let $G$ be a compact Abelian group with nonmetrizable connected component of zero. Then there exist $K$, an $\omega$-bounded (hence countably compact) dense subgroup of $G$, and a proper, totally dense subgroup $H$ of $G$ such that $K \subseteq H$ (in particular, $H$ is pseudocompact).

For weakly totally dense subgroups of compact groups the situation changes entirely.

1.10. Theorem. For a compact connected group $G$ the following are equivalent:

(i) $G$ contains a proper, weakly totally dense, countably compact subgroup;

(ii) $G$ contains a proper, weakly totally dense, $\omega$-bounded subgroup; and

(iii) the center $Z$ of $G$ is not a $G_{\delta}$-subgroup of $G$.

It follow from this theorem that if $G$ is a compact, connected, simple Lie group, then $G^{\omega_{1}}$ contains a proper, weakly totally dense, $\omega$-bounded (hence countably compact) subgroup. So in Theorem 1.4 "totally dense" cannot be replaced by "weakly totally dense."

In conclusion let us mention open problems left unanswered by our results. 
1.11. Problem. Does Theorem 1.8 hold without any additional set-theoretic assumptions beyond ZFC? Is it possible to drop "Abelian" in this theorem?

1.12. Problem. Let $G$ be a compact Abelian group without closed, torsion $G_{\delta^{-}}$ subgroups. Is it possible to find $K$, a dense, $\omega$-bounded (or at least, countably compact) subgroup of $G$, and a proper, totally dense subgroup $H$ of $G$ such that $K \subseteq H$ ?

1.13. Problem. When does a compact group contain a proper, weakly totally dense, countably compact ( $\omega$-bounded) subgroup?

In connection with the first part of 1.11 one should mention the following significant result of Comfort and Robertson [4]: the existence of a compact group $K$ with a totally dense, pseudocompact subgroup of properly smaller cardinality than $|K|$ is undecidable in ZFC.

\section{Preliminaries}

We denote by $\mathbb{N}$ and $\mathbb{Z}$ the sets of naturals and integers respectively, by $c$ the cardinality of the continuum, and by $\mathbb{T}$ the circle group.

2.1. Lemma. Let $G$ be a compact group, $\varphi: G \rightarrow G^{\prime}$ a continuous homomorphism of $G$ onto $G^{\prime}, H^{\prime}$ a subgroup of $G^{\prime}$ and $H=\varphi^{-1}\left(H^{\prime}\right)$. Then:

(i) if $H^{\prime}$ is dense in $G^{\prime}$, then $H$ is dense in $G$;

(ii) if $H^{\prime}$ is (weakly) totally dense in $G^{\prime}$, then $H$ is (weakly) totally dense in $G$;

(iii) if $H^{\prime}$ is pseudocompact, then so is $H$; and

(iv) if $H^{\prime}$ is $\omega$-bounded, then so is $H$.

Proof. For (i), (ii), and (iii) see [5, 4.1]. To prove (iv) take a countable subset $D$ of $H$. Since $H^{\prime}$ is $\omega$-bounded, there exists a compact subset $K$ of $H^{\prime}$ containing $\varphi(D)$. Then $D$ is contained in $\varphi^{-1}(K)$, which is compact as a preimage of a compact set under the perfect map $\varphi[9,3.7 .2]$.

2.2. Lemma. Let $G$ be a compact Abelian group and $C$ be the connected component of $G$. Then there exists a continuous surjective homomorphism $\varphi: G \rightarrow \mathbb{T}^{A}$ with totally disconnected kernel such that:

(a) $|A|$ coincides with the weight of $C$ if $C$ is not metrizable, and

(b) $A \neq \varnothing$ iff $C \neq\{0\}$.

Proof. Let $X$ be the (discrete) dual group of $G$. Consider a free subgroup $F$ of $G$ such that $X / F$ is torsion, and let $f: F \rightarrow X$ be the inclusion. Then $F$ is isomorphic to $\mathbb{Z}^{(A)}$ for some $A$ that is nonempty iff $X$ is nontorsion and satisfies $|A|=|F|=|X / t(X)|$ in case the latter group is uncountable. Since the dual group of $F$ is isomorphic to $\mathbb{T}^{A}$, taking the adjoint homomorphism $\varphi=$ $f^{*}$ we obtain a continuous surjective homomorphism $\varphi: G \rightarrow \mathbb{T}^{A}$, which has the desired properties. To verify this one should note that $\operatorname{ker} \varphi$ is isomorphic to the dual group of the quotient $X / f(F)$, while $C$ is isomorphic to the dual of $X / t(X)[11,24.11]$. Now use the fact that the dual group of a torsion group is totally disconnected $[11,24.26]$. On the other hand the second isomorphism yields both (a) and (b) in view of the above properties of $F$ and the equality $w(C)=|X / t(X)|[11,24.15]$; in particular, $C \neq\{0\}$ iff $X$ is not torsion, i.e. $A \neq \varnothing[11,24.26]$. 
A subset of an Abelian group $G$ is said to be independent if it generates a free subgroup of $G$.

2.3. Lemma. Each nonempty open subset of a nontorsion, compact Abelian group $G$ contains an independent subset of size $c$.

Proof. Let $W \neq \varnothing$ be an open subset of $G$. For $n \in \mathbb{N}$ set

$S_{n}=\left\{\left(k_{0}, \ldots, k_{n}\right) \in \mathbb{Z}^{n+1}:\left|k_{j}\right| \leq n\right.$ for all $j \leq n$ and $k_{i} \neq 0$ for some $\left.i \leq n\right\}$.

2.4. Claim. For each finite sequence $U_{0}, \ldots, U_{n}$ of nonempty open subsets of $G$ there exists a sequence $V_{0}, \ldots, V_{n}$ of nonempty open subsets of $G$ such that $V_{i} \subseteq U_{i}$ for $i \leq n$, and if $\left(k_{0}, \ldots, k_{n}\right) \in S_{n}$ and $x_{j} \in V_{j}$ for all $j \leq n$, then $k_{0} x_{0}+\cdots+k_{n} x_{n} \neq 0$.

Proof. Fix $\left(k_{0}, \ldots, k_{n}\right) \in S_{n}$. The map $\pi: G^{n+1} \rightarrow G$ defined by

$$
\pi\left(x_{0}, \ldots, x_{n}\right)=k_{0} x_{0}+\ldots+k_{n} x_{n}
$$

is a continuous homomorphism. Since $G$ is compact, $\pi$ is an open map onto the compact group $H=\pi(G)$, so the set $U=\pi\left(U_{0} \times \cdots \times U_{n}\right) \neq \varnothing$ is open in $H$. Since $\left(k_{0}, \ldots, k_{n}\right) \in S_{n}, k_{i} \neq 0$ for some $i \leq n$. If $z \in G$ is a nontorsion element, then so is $k_{i} z \in H$. Therefore $H$ is infinite, and hence $U \neq\{0\}$. For $i \leq n$ fix $y_{i} \in U_{i}$ so that $\pi\left(y_{0}, \ldots, y_{n}\right)=k_{0} y_{0}+\cdots+k_{n} y_{n} \neq 0$. By the continuity of $\pi$ we can find open sets $W_{0}, \ldots, W_{n}$ such that $y_{i} \in W_{i} \subseteq U_{i}$ for $i \leq n$ and $0 \notin \pi\left(W_{0} \times \cdots \times W_{n}\right)$. Since $S_{n}$ is finite, repeating this argument finitely many times provides finally $V_{0}, \ldots, V_{n}$.

For $f \in\{0,1\}^{\omega}$ and $n \in \mathbb{N}$ let $f \mid n$ denote the restriction of the function $f$ to $\{0, \ldots, n\}$. For $n \in \mathbb{N}$ set $\mathscr{F}_{n}=\left\{f \mid n: f \in\{0,1\}^{\omega}\right\}$, and let $\mathscr{F}=$ $\bigcup\left\{\mathscr{F}_{n}: n \in \mathbb{N}\right\}$. By recursion we can construct, applying Claim 2.4, a family $\left\{V_{f}: f \in \mathscr{F}\right\}$ of open subsets of $W$ such that:

(i) if $f, g \in \mathscr{F}$ are distinct then $V_{f} \cap V_{g}=\varnothing$;

(ii) if $g \in \mathscr{F}$ properly extends $f \in \mathscr{F}$ then $\bar{V}_{g} \subseteq V_{f}$; and

(iii) for all $n \in \mathbb{N}$ and $F \subseteq \mathscr{F}_{n}$, if $k_{f} \in \mathbb{Z}$ with $\left|k_{f}\right| \leq 2^{n}$ and $x_{f} \in V_{f}$ for each $f \in F$ are fixed so that $\sum\left\{k_{f} x_{f}: f \in F\right\}=0$, then $k_{f}=0$ for all $f \in F$.

Because $G$ is compact, for each $f \in\{0,1\}^{\omega}$ we can pick a point $y_{f} \in$ $\bigcap\left\{\bar{V}_{f \mid n}: n \in \mathbb{N}\right\}$. From (i) and (ii) it follows that $y_{f^{\prime}} \neq y_{f^{\prime \prime}}$ whenever $f^{\prime}, f^{\prime \prime} \in\{0,1\}^{\omega}$ are distinct, so $Y=\left\{y_{f}: f \in\{0,1\}^{\omega}\right\} \subseteq W$ has size $c$. Now (iii) implies that $Y$ is independent.

2.5. Definition. Let $\left\{G_{\alpha}: \alpha \in A\right\}$ be a family of topological groups. For $g=\left\{g_{\alpha}\right\}_{\alpha \in A} \in G=\Pi\left\{G_{\alpha}: \alpha \in A\right\}$ let supp $g=\left\{\alpha \in A: g_{\alpha} \neq e\right\}$. Define $\Sigma\left\{G_{\alpha}: \alpha \in A\right\}=\{g \in G:|\operatorname{supp} g| \leq \omega\}$ and $\sigma\left\{G_{\alpha}: \alpha \in A\right\}=\{g \in G: \operatorname{supp} g$ is finite $\}$.

2.6. Lemma [1, Corollary 6.11]. If $A$ is uncountable and $\left\{G_{\alpha}: \alpha \in A\right\}$ is a family consisting of compact groups, then $\Sigma\left\{G_{\alpha}: \alpha \in A\right\}$ is a proper, dense, $\omega$-bounded subgroup of $G=\Pi\left\{G_{\alpha}: \alpha \in A\right\}$.

\section{TOTALLY DENSE, COUNTABLY COMPACT SUBGROUPS}

3.1. Lemma. Let $G$ be a compact group, $H$ its totally dense, countably compact subgroup, and $K$ a closed, totally disconnected subgroup of $G$. Then $K \subseteq H$. 
Proof. Pick $x \in K$, and let $N$ be the smallest closed subgroup of $G$ that contains $x$. Since $N \subseteq K, N$ is totally disconnected, so metrizable by [11, 25.16]. Since $H$ is totally dense in $G, N^{*}=H \cap N$ is dense in $N$. Being a closed subspace of $H, N^{*}$ is countably compact. Since countably compact metrizable spaces are compact $[9,4.1 .17]$, we conclude that $N^{*}$ is compact, and so $N^{*}=N$ in view of the density of $N^{*}$ in $N$. Therefore $x \in N \subseteq H$.

Proof of Theorem 1.4. Let $G$ be an $\omega$-bounded group and $H$ be a totally dense, countably compact subgroup of $G$. Pick arbitrary $x \in G$. To prove our theorem it suffices to verify that $x \in H$. Let $N$ be the smallest closed subgroup of $G$ containing $x$. Then $N$ is Abelian, because it contains the cyclic (hence Abelian) subgroup generated by $x$ as a dense subgroup. Since $G$ is $\omega$-bounded, $N$ is compact. By the total density of $H$ in $G, H^{\prime}=H \cap N$ is a totally dense subgroup of $N$. Being a closed subspace of $H, H^{\prime}$ is countably compact. If $N$ is totally disconnected, then $x \in N \subseteq H^{\prime} \subseteq H$ by Lemma 3.1 applied to $N, H^{\prime}$, and $N$ instead of $G, H$, and $K$ respectively. Otherwise Lemma 2.2 yields that there exist a nonempty set $A$ and a continuous surjective homomorphism $\varphi: N \rightarrow \mathbb{T}^{A}$ with totally disconnected kernel $K$.

\subsection{Claim. $\varphi\left(H^{\prime}\right)=\mathbb{T}^{A}$.}

Proof. One easily checks that $\varphi\left(H^{\prime}\right)$ is totally dense in $\mathbb{T}^{A}$. Clearly this subgroup is also countably compact. Let $\mathbb{Q} / \mathbb{Z}$ be the torsion subgroup of $\mathbb{T}$ and $x \in(\mathbb{Q} / \mathbb{Z})^{A}$. Then each coordinate $x_{\alpha}$ of $x$ lies in a finite subgroup $F_{\alpha}$ of $\mathbb{T}$, so $x \in F=\Pi\left\{F_{\alpha}: \alpha \in A\right\} \subseteq \mathbb{T}^{A}$. Since $F$ is a closed, totally disconnected subgroup of $\mathbb{T}^{A}$, from Lemma 3.1 it follows that $x \in F \subseteq \varphi\left(H^{\prime}\right)$. Therefore $(\mathbb{Q} / \mathbb{Z})^{A} \subseteq \varphi\left(H^{\prime}\right)$. Now fix $z=\left\{z_{\alpha}\right\}_{\alpha \in A} \in \mathbb{T}^{A}$. For every $\alpha \in A$ choose a sequence $\left\{y_{\alpha}^{n}\right\}_{n=1}^{\infty}$ of distinct points of $\mathbb{Q} / \mathbb{Z}$ converging to $z_{\alpha}$. For $n \in \mathbb{N}$ define $y^{n}=\left\{y_{\alpha}^{n}\right\}_{\alpha \in A} \in(\mathbb{Q} / \mathbb{Z})^{A}$ and note that $\left\{y^{n}: n \in \mathbb{N}\right\} \subseteq(\mathbb{Q} / \mathbb{Z})^{A} \subseteq \varphi\left(H^{\prime}\right)$ is a sequence converging to $z$. Since $\varphi\left(H^{\prime}\right)$ is countably compact, $z \in \varphi\left(H^{\prime}\right)$. This yields $\varphi\left(H^{\prime}\right)=\mathbb{T}^{A}$.

Since $K$ is a closed, totally disconnected subgroup of $N, K \subseteq H^{\prime}$ by Lemma 3.1. This, together with Claim 3.2, implies that

$$
x \in N=H^{\prime}+K \subseteq H^{\prime}+H^{\prime}=H^{\prime} \subseteq H .
$$

Proof of Theorem 1.10. (i) $\rightarrow$ (iii). Assume that $Z$ is a $G_{\delta}$-subset of $G$. Let $H$ be a weakly totally dense, countably compact subgroup of $G$ and $\pi$ : $G \rightarrow G / Z$ be the quotient homomorphism. Then $\pi(H)$ is a dense, countably compact subgroup of the metrizable group $G / Z[11,8.6]$. Since countably compact metrizable spaces are compact [9, 4.1.17], $\pi(H)=G / Z$. Further, since every subgroup of $Z$ is normal in $G$ and $H$ is weakly totally dense in $G$, we conclude that $H^{\prime}=H \cap Z$ is a weakly totally dense subgroup of $Z$. Since $Z$ is Abelian, $H^{\prime}$ is totally dense in $Z$. Being a closed subspace of $H$, $H^{\prime}$ is countably compact. From Theorem 1.4 it follows that $Z=H^{\prime} \subseteq H$. Therefore $G=Z+H \subseteq H+H=H$.

(iii) $\rightarrow$ (ii). If $Z$ is not a $G_{\delta}$-subset of $G$, then the quotient $L=G / Z$ is not metrizable. Moreover, $L=\Pi\left\{L_{\alpha}: \alpha \in A\right\}$, where $A$ is uncountable and each $L_{\alpha}$ is a simple, connected, compact Lie group [14]. Define $L^{\prime}=$ 
$\Sigma\left\{L_{\alpha}: \alpha \in A\right\} \subseteq L$ and $H=\varphi^{-1}\left(L^{\prime}\right)$, where $\varphi: G \rightarrow L$ is the quotient homomorphism. By Lemma 2.6, $L^{\prime}$ is a proper, $\omega$-bounded subgroup of $L$, and so is $H$ (Lemma 2.1 (iv)). From [8] it follows that $K=\sigma\left\{L_{\alpha}: \alpha \in A\right\}$ is a totally minimal group, so $K$ is weakly totally dense in $L$ by 1.3 . Since $K \subseteq L^{\prime}, L^{\prime}$ is also weakly totally dense in $L$. Now Lemma 2.1(ii) implies that $H$ is weakly totally dense in $G$.

(ii) $\rightarrow$ (i) is trivial.

\section{REDUCTION PRINCIPLE}

In this section we prove a general statement that permits us to construct homomorphisms of groups that lower the weight of groups and preserve some of their properties.

4.1. Reduction principle. Let $\mathscr{G}$ be a class of topological groups such that:

(i) if $G \in \mathscr{G}$ and $\pi: G \rightarrow H$ is a continuous surjective homomorphism, then $H \in \mathscr{G}$.

Suppose also that for each $G \in \mathscr{G}$ a family $\mathscr{N}(G)$ of $G_{\delta}$-subgroups of $G$ is assigned in such a way that the following conditions hold:

(ii) if $G \in \mathscr{G}, \pi: G \rightarrow H$ is a continuous surjective homomorphism and $N \in \mathscr{N}(H)$, then $\pi^{-1}(N) \in \mathscr{N}(G)$; and

(iii) if $B$ is a $G_{\delta}$-subset of $G \in \mathscr{G}$ that contains the neutral element of $G$, then there exists $N \in \mathscr{N}(G)$ with $N \subseteq B$.

Further, let $Q$ be a class of topological groups having the following properties:

(iv) if $\pi: G \rightarrow H$ is a continuous surjective homomorphism and $H \in Q$, then $G \in Q$;

(v) if $N$ is a subgroup of a topological group $G$ and $N \in Q$, then $G \in Q$; and

(vi) if $G \in \mathscr{G}$ and $N \in \mathscr{N}(G) \cap Q$, then there exists a continuous homomorphism $\pi: G \rightarrow H$ onto a topological group $H$ of weight $\leq \omega_{1}$ so that $\pi(N) \in Q$.

Finally suppose that $G \in \mathscr{G}$ is such that $N \in \mathcal{N}(G)$ implies $N \in Q$. Then there exists a continuous homomorphism $\varphi: G \rightarrow H$ of $G$ onto a topological group $H$ of weight $\leq \omega_{1}$ such that each $G_{\delta}$-subgroup of $H$ belongs to $Q$.

Proof. Let us start by verifying the following

4.2. Claim. Assume that $\pi: G \rightarrow H$ is a continuous homomorphism onto a topological group $H$ of weight $\leq \omega_{1}$. Then there exists a topological group $\widetilde{H}$ of weight $\leq \omega_{1}$ and continuous homomorphisms $\varphi: G \rightarrow \widetilde{H}, \psi: \widetilde{H} \rightarrow H$ such that $\pi=\psi \circ \varphi$ and $\psi^{-1}(N) \in Q$ whenever $N \in \mathscr{N}(H)$.

Proof. Observe that $G \in \mathscr{G}$ and (i) imply $H \in \mathscr{G}$. Fix a base $\left\{U_{\beta}: \beta<\omega_{1}\right\}$ of open neighbourhoods of the neutral element of $H$. For $\alpha<\omega_{1}$ apply (iii) to choose $N_{\alpha} \in \mathscr{N}(H)$ with $N_{\alpha} \subseteq \cap\left\{U_{\beta}: \beta<\alpha\right\}$, and let $N_{\alpha}^{*}=\pi^{-1}\left(N_{\alpha}\right)$. Then $N_{\alpha}^{*} \in \mathscr{N}(G)$ by (ii), and so $N_{\alpha}^{*} \in Q$ by our assumption on $G$. Use (vi) to find a continuous homomorphism $\varphi_{\alpha}: G \rightarrow H_{\alpha}$ onto a topological group $H_{\alpha}$ of weight $\leq \omega_{1}$ so that $\varphi_{\alpha}\left(N_{\alpha}^{*}\right) \in Q$. Let $\varphi: G \rightarrow \widetilde{H}=H \times$ $\Pi\left\{H_{\alpha}: \alpha<\omega_{1}\right\}$ be the continuous homomorphism that sends each $g \in G$ to the point $\varphi(g)=\left(\pi(g),\left\{\varphi_{\alpha}(g)\right\}_{\alpha<\omega_{1}}\right)$, and let $\psi_{\alpha}: \widetilde{H} \rightarrow H_{\alpha}$ and $\psi: \widetilde{H} \rightarrow H$ 
be the canonical projections. Then we have the following commutative diagram:

$$
\begin{array}{ccc}
G & \stackrel{\pi}{\rightarrow} & H \\
\varphi_{\alpha} \downarrow & \searrow \varphi & \uparrow \psi \\
H_{\alpha} & \overleftarrow{\psi_{\alpha}} & \widetilde{H} .
\end{array}
$$

It is clear that $\widetilde{H}$ has weight $\leq \omega_{1}$. To establish the last property in the conclusion of our claim we pick $N \in \mathscr{N}(H)$. Let $N=\bigcap\left\{V_{n}: n \in \mathbb{N}\right\}$, where $V_{n}$ 's are open in $H$. For each $n \in \mathbb{N}$ choose $\alpha_{n}<\omega_{1}$ with $U_{\alpha_{n}} \subseteq V_{n}$, and set $\alpha=\sup \left\{\alpha_{n}: n \in \mathbb{N}\right\}$. By our construction $\varphi_{\alpha}\left(N_{\alpha}^{*}\right)=\psi_{\alpha}\left(\varphi\left(N_{\alpha}^{*}\right)\right)$ belongs to $Q$. Applying (iv) we conclude that $\varphi\left(N_{\alpha}^{*}\right)=\varphi\left(\pi^{-1}\left(N_{\alpha}\right)\right)=\psi^{-1}\left(N_{\alpha}\right)$ belongs to $Q$. Since

$$
\begin{array}{r}
N_{\alpha} \subseteq \bigcap\left\{U_{\beta}: \beta<\alpha\right\} \subseteq \bigcap\left\{V_{n}: n \in \mathbb{N}\right\}=N, \\
\psi^{-1}\left(N_{\alpha}\right) \subseteq \psi^{-1}(N) \text {, and so } \psi^{-1}(N) \in Q \text { by (v). } \square
\end{array}
$$

Now Claim 4.2 permits us to construct by transfinite recursion a well-ordered inverse spectrum $\mathbf{S}=\left\{H_{\alpha}, \psi_{\beta}^{\alpha}, \beta<\alpha<\omega_{1}\right\}$ and maps $\varphi_{\alpha}: G \rightarrow H_{\alpha}$ for all $\alpha<\omega_{1}$ such that:

(a) all $H_{\alpha}$ 's are topological groups of weight $\leq \omega_{1}$;

(b) $\varphi_{\alpha}: G \rightarrow H_{\alpha}$ and $\psi_{\beta}^{\alpha}: H_{\alpha} \rightarrow H_{\beta}$ are continuous surjective homomorphisms whenever $\beta<\alpha<\omega_{1}$ :

(c) $\varphi_{\beta}=\psi_{\beta}^{\alpha} \circ \varphi_{\alpha}$ provided that $\beta<\alpha<\omega_{1}$; and

(d) if $\alpha<\omega_{1}$ and $N \in \mathscr{N}\left(H_{\alpha}\right)$, then $\left(\psi_{\alpha}^{\alpha+1}\right)^{-1}(N) \in Q$.

To begin with let $\varphi_{\circ}: G \rightarrow H_{\circ}=\{e\}$ be the trivial homomorphism. For a nonlimit ordinal Claim 4.2 works. For $\alpha$ a limit ordinal let $K=\Pi\left\{H_{\beta}: \beta<\right.$ $\alpha\}$ and let $\varphi_{\alpha}: G \rightarrow K$ be the map sending $g \in G$ to $\varphi_{\alpha}(g)=\left\{\varphi_{\beta}(g)\right\}_{\beta<\alpha}$. Then $H_{\alpha}=\varphi_{\alpha}(G)$ is as required.

Now $H$, the limit of the spectrum $\mathbf{S}$, has weight $\leq \omega_{1}$. Moreover, there exists the continuous homomorphism $\varphi: G \rightarrow H$ such that $\varphi_{\alpha}=\psi_{\alpha} \circ \varphi$ for all $\alpha<\omega_{1}$, where $\psi_{\alpha}: H \rightarrow H_{\alpha}$ are the limit projections of $\mathbf{S}$. Let us show that $H$ is as required. Fix $N$, a $G_{\delta}$-subgroup of $H$. Let $N=\bigcap\left\{V_{n}: n \in \mathbb{N}\right\}$, where all $V_{n}$ 's are open in $H$. For $n \in \mathbb{N}$ choose $\alpha_{n}<\omega_{1}$ and $U_{n}$, an open neighbourhood of the neutral element of $H_{\alpha_{n}}$, so that $\psi_{\alpha_{n}}^{-1}\left(U_{n}\right) \subseteq V_{n}$. Define $\alpha=\sup \left\{\alpha_{n}: n \in \mathbb{N}\right\}$ and $W_{n}=\left(\psi_{\alpha_{n}}^{\alpha}\right)^{-1}\left(U_{n}\right)$. Since $\varphi_{\alpha}: G \rightarrow H_{\alpha}$ is a continuous surjective homomorphism by (b), from $G \in \mathscr{G}$ and (i) it follows that $H_{\alpha} \in \mathscr{G}$; we can use (iii) to find $N_{\alpha} \in \mathscr{N}\left(H_{\alpha}\right)$ with $N_{\alpha} \subseteq \bigcap\left\{W_{n}: n \in \mathbb{N}\right\}$. Then

$$
\begin{aligned}
\psi_{\alpha}^{-1}\left(N_{\alpha}\right) & \subseteq \bigcap\left\{\psi_{\alpha}^{-1}\left(W_{n}\right): n \in \mathbb{N}\right\} \\
& =\bigcap\left\{\psi_{\alpha_{n}}^{-1}\left(U_{n}\right): n \in \mathbb{N}\right\} \subseteq \bigcap\left\{V_{n}: n \in \mathbb{N}\right\}=N .
\end{aligned}
$$

From (d) it follows that $\left(\psi_{\alpha}^{\alpha+1}\right)^{-1}\left(N_{\alpha}\right) \in Q$, so

$$
\psi_{\alpha}^{-1}\left(N_{\alpha}\right)=\psi_{\alpha+1}^{-1}\left(\left(\psi_{\alpha}^{\alpha+1}\right)^{-1}\left(N_{\alpha}\right)\right) \in Q
$$

by (iv). Now (*) and (v) yield $N \in Q$.

4.3. Corollary. If $G$ is a compact group so that none of its normal, closed $G_{\delta^{-}}$ subgroups is torsion, then there exists a continuous homomorphism $\pi: G \rightarrow H$ of $G$ onto a topological group $H$ of weight $\leq \omega_{1}$ having no torsion $G_{\delta}$-subgroups. 
Proof. Let $\mathscr{G}$ be the class of all compact groups, $Q$ the class of nontorsion topological groups, and for every $G \in \mathscr{G}$ let $\mathscr{N}(G)$ be the set of all closed, normal $G_{\delta}$-subgroups of $G$. Then properties (i), (ii), (iv), and (v) of 4.1 can be verified trivially. Property (iii) follows from [11, 8.7]. To check (vi) let $N$ be a nontorsion subgroup of $G$. Fix a nontorsion element $x \in N$. Since $G$ is compact, for each $n \in \mathbb{N}$ there exists a continuous homomorphism $\pi_{n}: G \rightarrow K_{n}$ into a compact metrizable group $K_{n}$ such that $\pi_{n}(n x) \neq e$ [11, 22.12]. If $\pi: G \rightarrow K=\Pi\left\{K_{n}: n \in \mathbb{N}\right\}$ sends each $g \in G$ to the point $\pi(g)=\left\{\pi_{n}(g)\right\}_{n \in \mathbb{N}}$ and $H=\pi(G)$, then $\pi: G \rightarrow H$ is a continuous homomorphism onto a compact metrizable group $H$ so that $\pi(N) \in Q$. Now to finish the proof apply the Reduction principle.

\section{TOTALly DENSE, PSEUdOCOMPACT SUBGROUPS}

For $g \in G$ we denote by $\langle g\rangle$ the subgroup of $G$ generated by $g$.

5.1. Lemma. Suppose that $G$ is a compact Abelian group, $N$ is its closed, nontorsion subgroup, $U$ is an open subset of $G, U \cap N \neq \varnothing, X \subseteq G$, and $|X|<c$. Then there exists a nontorsion element $h \in U \cap N$ so that $\langle h\rangle \cap X \subseteq\{0\}$.

Proof. By Lemma 2.3 there exists an independent set $S \subseteq U \cap N$ with $|S|=c$. Since $S$ is independent, for $T=\{g \in S:\langle g\rangle \cap(X \backslash\{0\}) \neq \varnothing\}$ we would have $|T| \leq|X|<c$, and now it suffices to pick $h \in S \backslash T$.

5.2. Lemma. Let $G$ be a compact Abelian group of size $c$ having no closed, torsion $G_{\delta}$-subgroups. Then $G$ contains a proper, totally dense, pseudocompact subgroup.

Proof. Since $G$ is compact and $|G|=c$, we can fix a base $\mathscr{B}=\left\{U_{\alpha}: \alpha<c\right\}$ of $G[9,3.1 .21]$ and enumeration $\left\{x_{\alpha}: \alpha<c\right\}$ of all points of $G$. Fix $F$, a closed $G_{\delta}$-subset of $G$, and let $F=\bigcap\left\{V_{n}: n \in \mathbb{N}\right\}$, where each $V_{n}$ is open is $G$. Since $F$ is compact and $\mathscr{B}$ is a base of $G$, for every $n \in \mathbb{N}$ there exists a finite collection $\mathscr{C}(F, n) \subseteq \mathscr{B}$ with $F \subseteq \bigcup \mathscr{C}(F, n) \subseteq V_{n}$. This assigns to each closed $G_{\delta}$-subset $F$ of $G$ a sequence $\mathscr{S}_{F}=\{\mathscr{C}(F, n): n \in \mathbb{N}\}$ of finite subcollections of $\mathscr{B}$. Observe that $F \subseteq \bigcap\{\bigcup \mathscr{C}(F, n): n \in \mathbb{N}\} \subseteq$ $\bigcap\left\{V_{n}: n \in \mathbb{N}\right\}=F$, so $\mathscr{S}_{F^{\prime}} \neq \mathscr{S}_{F^{\prime \prime}}$ for different $F^{\prime}$ and $F^{\prime \prime}$. Since there are at most $c$ such sequences, this argument shows that there exist at most $c$ closed $G_{\delta}$-subgroups of $G$; so let $\left\{L_{\alpha}: \alpha<c\right\}$ enumerate all such subgroups. For $\alpha<c$ let $N_{\alpha}$ be the smallest closed subgroup of $G$ that contains $x_{\alpha}$. Let $\mathscr{E}=\{\alpha<c: \alpha$ is even $\}$ and $\mathscr{O}=\{\alpha<c: \alpha$ is odd $\}$. (Recall that a nonlimit ordinal $\beta+n$, where $\beta$ is a limit ordinal and $n \in \mathbb{N}$, is said to be even (odd) if $n$ is even (odd).) Fix functions $\varphi: c \rightarrow c$ and $\psi: c \rightarrow c$ having the following property:

$$
\begin{aligned}
& \text { if } \alpha, \beta<c, \text { then there are } \lambda \in \mathscr{E} \text { and } \mu \in \mathscr{O} \\
& \text { with } \varphi(\lambda)=\varphi(\mu)=\alpha \text { and } \psi(\lambda)=\psi(\mu)=\beta
\end{aligned}
$$

Since $G$ is not torsion, we can choose an infinite cyclic subgroup $Z$ of $G$.

Now by transfinite recursion we construct an increasing chain $\left\{H_{\gamma}: \gamma<c\right\}$ of subgroups of $G$ such that for all $\gamma<c$ the following conditions are satisfied:

$\left(\mathrm{i}_{\gamma}\right) \quad\left|H_{\gamma}\right|<c$ 
(ii $i_{\gamma}$ if $\gamma \in \mathscr{O}$ and $U_{\varphi(\gamma)} \cap N_{\psi(\gamma)} \neq \varnothing$, then $U_{\varphi(\gamma)} \cap H_{\gamma} \cap N_{\psi(\gamma)} \neq \varnothing$;

(iii $\left.{ }_{\gamma}\right) H_{\gamma} \cap\left(x_{\varphi(\gamma)}+L_{\psi(\gamma)}\right) \neq \varnothing$ for $\gamma \in \mathscr{E}$;

(iv $\left.{ }_{\gamma}\right) H_{\gamma} \cap Z=\{0\}$.

To start the recursion set $H_{-1}=\{0\}$ for convenience. Then suppose that $\gamma<c$ and that $H_{\lambda}$ satisfying $\left(\mathrm{i}_{\lambda}\right)-\left(\mathrm{iv}_{\lambda}\right)$ have already been defined for $\lambda<\gamma$. Let us define $H_{\gamma}$.

Case 1. If $\gamma$ is a limit ordinal, then $H_{\gamma}=\bigcup\left\{H_{\lambda}: \lambda<\gamma\right\}$ works.

Case 2. $\gamma=\lambda+1 \in \mathscr{O}$. If $U_{\varphi(\gamma)} \cap N_{\psi(\gamma)}=\varnothing$, then it suffices to set $H_{\gamma}=H_{\lambda}$. Assume that $U_{\varphi(\gamma)} \cap N_{\psi(\gamma)} \neq \varnothing$ and consider two cases.

(a) $x_{\psi(\gamma)} \in G$ is torsion. In this case define $H_{\gamma}=H_{\gamma}+\left\langle x_{\psi(\gamma)}\right\rangle$. Observe that $H_{\gamma} \cap N_{\psi(\gamma)}=N_{\psi(\gamma)}$; in particular, $U_{\varphi(\gamma)} \cap H_{\gamma} \cap N_{\psi(\gamma)}=U_{\varphi(\gamma)} \cap N_{\psi(\gamma)} \neq \varnothing$. If (iv $\gamma$ ) fails, then $g+k x_{\psi(\gamma)}=z$ for some $g \in H_{\lambda}, k \in \mathbb{N}$, and $z \in Z, z \neq 0$. Since $x_{\psi(\gamma)}$ is torsion, there is $n \in \mathbb{N}$ with $n x_{\psi(\gamma)}=0$, so

$$
0 \neq n z=n\left(g+k x_{\psi(\gamma)}\right)=n g+k n x_{\psi(\gamma)}=n g,
$$

a contradiction with $\left(\operatorname{iv}_{\lambda}\right)$.

(b) $x_{\psi(\gamma)} \in G$ is not torsion. Then $N_{\psi(\gamma)}$ is not torsion, and since $\mid H_{\lambda}+$ $Z \mid<c$ by $\left(\mathrm{i}_{\lambda}\right)$, we can use Lemma 5.1 to choose $h \in U_{\varphi(\gamma)} \cap N_{\psi(\gamma)}$ with $\langle h\rangle \cap\left(H_{\lambda}+Z\right)=\{0\}$. From the last equality and $\left(\mathrm{iv}_{\lambda}\right)$ it follows that $H_{\gamma}=$ $H_{\lambda}+\langle h\rangle$ satisfies $\left(\mathrm{iv}_{\gamma}\right)$. It is also clear that $h \in U_{\varphi(\gamma)} \cap H_{\gamma} \cap N_{\psi(\gamma)}$.

Case 3. $\gamma=\lambda+1 \in \mathscr{E}$. Since $L_{\psi(\gamma)}$ is a $G_{\delta}$-subgroup of $G$, the assumption of our lemma yields that $L_{\psi(\gamma)}$ is not torsion. Since $\left|H_{\lambda}+Z+\left\langle x_{\varphi(\gamma)}\right\rangle\right|<c$ by $\left(i_{\lambda}\right)$, there exists a nontorsion element $h \in L_{\psi(\gamma)}$ with $\langle h\rangle \cap\left(H_{\lambda}+Z+\left\langle x_{\psi(\gamma)}\right\rangle\right)=$ \{0\} (Lemma 5.1). Now note that $H_{\gamma}=H_{\lambda}+\left\langle x_{\varphi(\gamma)}+h\right\rangle$ is as required.

5.3. Claim. $H=\cup\left\{H_{\gamma}: \gamma<c\right\}$ is a proper, totally dense, pseudocompact subgroup of $G$.

Proof. From $\left(\mathrm{iv}_{\gamma}\right)$ for all $\gamma<c$ it follows that $H \cap Z=\{0\}$, i.e. $H$ is a proper subgroup of $G$. To show that $H$ is totally dense in $G$, suppose that $U$ is open in $G, N$ is a closed subgroup of $G$, and $U \cap N \neq \varnothing$. Then $x_{\beta} \in U \cap N$ for some $\beta<c$. Since $\mathscr{B}$ is a base of $G, x_{\beta} \in U_{\alpha} \subset U$ for some $\alpha<c$. Use $(* *)$ to find $\gamma \in \mathscr{O}$ with $\varphi(\gamma)=\alpha$ and $\psi(\gamma)=\beta$. Then (ii ${ }_{\gamma}$ ) yields $U \cap H \cap N \supseteq U_{\alpha} \cap H_{\gamma} \cap N_{\beta} \neq \varnothing$. Therefore $H \cap N$ is dense in $N$, and so $H$ is totally dense in $G$.

Since $H$ is dense in the compact group $G, G$ is the completion of $H$. Now pick $x \in G$ and a closed $G_{\delta}$-subgroup $L$ of $G$. Choose $\alpha, \beta<c$ so that $x=x_{\alpha}$ and $L=L_{\beta}$. Use $(* *)$ to find $\gamma \in \mathscr{E}$ with $\varphi(\gamma)=\alpha$ and $\psi(\gamma)=\beta$. Then $\left(\right.$ iii $\left._{\gamma}\right)$ implies $H \cap(x+L) \supseteq H_{\gamma} \cap\left(x_{\varphi(\gamma)}+L_{\psi(\gamma)}\right) \neq \varnothing$. Now the pseudocompactness of $H$ follows from [4, 1.2].

Proof of Theorem 1.8. (i) $\rightarrow$ (ii) follows from Theorem 1.7.

(ii) $\rightarrow$ (i). Use Corollary 4.3 to find a continuous homomorphism $\varphi$ : $G \rightarrow G^{\prime}$ of $G$ onto a compact group $G^{\prime}$ of weight $\leq \omega_{1}$ that has no torsion $G_{\delta}$-subgroups. Observe that $\left|G^{\prime}\right| \geq c$, because $G^{\prime}$ is a compact space without isolated points. On the other hand, since $G^{\prime}$ has weight $\leq \omega_{1},\left|G^{\prime}\right| \leq 2^{\omega_{1}}[9$, 1.5.1]. Since $2^{\omega_{1}}=c,\left|G^{\prime}\right|=c$. Let $H^{\prime}$ be a proper, totally dense, pseudocompact subgroup of $G^{\prime}$ which exists by Lemma 5.2. Then from Lemma 2.1(ii), (iii) it follows that $H=\varphi^{-1}\left(H^{\prime}\right)$ is a proper, totally dense, pseudocompact subgroup of $G$. 
Proof of Theorem 1.9. Use Lemma 2.2 to find a continuous surjective homomorphism $\varphi: G \rightarrow \mathbb{T}^{\omega_{1}}$. In $[5,5.1]$ a proper, totally dense subgroup $H^{\prime}$ of $\mathbb{T}^{\omega_{1}}$ with $\Sigma \mathbb{T}^{\omega_{1}} \subseteq H^{\prime}$ was constructed. Set $K=\varphi^{-1}\left(\Sigma \mathbb{T}^{\omega_{1}}\right)$ and $H=\varphi^{-1}\left(H^{\prime}\right)$. Then $K$ is a dense, $\omega$-bounded subgroup of $G$ (Lemmas 2.6 and 2.1(i), (iv)) and $H$ is a totally dense subgroup of $G$ (Lemma 2.1(ii)). Properness of $H$ and the inclusion $K \subseteq H$ are obvious.

\section{ACKNOWLEDGMENT}

The authors thank the referee for his valuable remarks.

\section{REFERENCES}

1. W. W. Comfort, Topological groups, Handbook of Set-theoretic Topology (K. Kunen and J. E. Vaughan, eds.) North-Holland, Amsterdam, New York, and Oxford, 1984, pp. 11431263.

2. W. W. Comfort and D. L. Grant, Cardinal invariants, pseudocompactness and minimality: some recent advances in the topological theory of topological groups, Topology Proc. 6 (1981), 227-265.

3. W. W. Comfort and L. C. Robertson, Cardinality constraints for pseudocompact and for totally dense subgroups of compact topological groups, Pacific J. Math. 119 (1985), 265285.

4. W. W. Comfort and K. A. Ross, Pseudocompactness and uniform continuity in topological groups, Pacific J. Math. 16 (1966), 483-496.

5. W. W. Comfort and T. Soundararajan, Pseudocompact group topologies and totally dense subgroups, Pacific J. Math. 100 (1982), 61-84.

6. D. Dikranjan and Iv. Prodanov, Totally minimal topological groups, Annuaire Univ. Sofia Fac. Math. Méc. 69 (1974/75), 5-11.

7. D. Dikranjan, Iv. Prodanov, and L. Stoyanov, Topological groups: characters, dualities and minimal group topologies, Pure Appl. Math., vol. 130, Marcel Dekker, New York and Basel, 1989.

8. V. Eberhardt and U. Schwanengel, $(\mathbb{Q} / \mathbb{Z})^{\mathbf{N}}$ est un groupe topologique minimal, Rev. Roumaine Math. Pures Appl. 27 (1982), 957-964.

9. R. Engelking, General topology, Warszawa, PWN, 1977.

10. I. I. Guran, On minimal topological groups, in Topology and Set Theory, Udmurt State Univ., Izhevsk, 1982, pp. 64-71. (Russian)

11. E. Hewitt and K. Ross, Abstract harmonic analysis. I, Springer-Verlag, Berlin, Heidelberg, and New York, 1963.

12. K. Kunen, Set theory. An introduction to independence proofs, Stud. Logic Foundations Math., vol. 102, North-Holland, Amsterdam, New York, and Oxford, 1980.

13. T. Soundararajan, Totally dense subgroups of topological groups, General Topology and Its Relations to Modern Analysis and Algebra III, Proc. Kanpur Topological Conf. 1968, Academia, Prague, 1971, pp. 299-300.

14. N. Th. Varopoulos, A theorem on the continuity of homomorphisms of locally compact groups, Proc. Cambridge Philos. Soc. 60 (1964), 449-463.

Institute of Mathematics, Bulgarian Academy of Sciences, 1090 Sofia, Bulgaria

Department of Mechanics and Mathematics, Moscow State University, 119899 Moscow, USSR 